\title{
Posición condílea según biotipo facial en tomografía de haz de cono
}

Condylar position according to facial biotype in cone beam tomography

Posição condilar de acordo com a biótipo facial em tomografia feixe cone

Melissa Marín-Estrada ${ }^{1 凶}$, Yenni Franco-Valencia ${ }^{1 凶}$, German Puerta-Salazar ${ }^{2}{ }^{凶}$, Bruno GutierrezQuiceno ${ }^{3}$

${ }^{1}$ Estudiante de $X$ semestre, Universidad del Valle.

${ }^{2}$ Ortodoncista. Docente de la Universidad del Valle.

${ }^{3}$ Odontólogo, Epidemiólogo, PhD(C) Salud Pública. Docente de la Universidad del Valle.

Fecha correspondencia:

Recibido: diciembre de 2020.

Aprobado: septiembre de 2021

Forma de citar:

Marín-Estrada M, Franco-

Valencia Y, Puerta-Salazar G,

Gutierrez-Quiceno B. Posición

condílea según biotipo facial en

tomografía de haz de cono. Rev.

CES Odont 2021; 34(2): 93-106

https://doi.org/10.21615/

cesodon.5998

Open access

(C) Derecho de autor

Licencia creative commons

Ética de publicaciones

Revisión por pares

Gestión por Open Journal System DOI: $10.21615 /$ cesodon. 5998

ISSNe 2215-9185

ISSN 0120-971X

Publica con nosotros

\section{Resumen}

Introducción y objetivo: La posición condilar, como el biotipo facial es importante para mantener una oclusión y un sistema estomatognático equilibrado. El objetivo del presente artículo es relacionar el biotipo facial con la posición condílea en tomografía de haz de cono, en pacientes sin reporte de trastornos temporomandibulares. Materiales y métodos: Se estudiaron 59 tomografías de pacientes 23 de sexo masculino y 36 femenino, con edades entre 14 y 59 años, se clasificaron en dolicofacial, mesofacial y braquifacial. Con el fin de evaluar la posición condilar se estudió la dimensión de los espacios articulares. Las tomografías fueron medidas con I-Cat vision y para el análisis estadístico se utilizó STATA 14, fue avalado por el comité de ética de la Universidad del Valle. Resultados: Se realizó correlación interobservador obteniendo un Kappa promedio de 0,85. 45 pacientes corresponden al biotipo braquifacial, 8 dolicofacial y 6 mesofacial. En todos los espacios articulares, los braquifaciales presentaron valores de mayor dimensión y el dolicofacial menor dimensión. Se encontró que los espacios mediales (CMS) presentaron valores similares en cuanto a lateralidad dentro de 
cada biotipo, teniendo diferencias de 0,02 a 0,09 sin embargo, para los espacios articulares centrales (CCS) y laterales (CLS) se observaron mayores diferencias entre un lado y otro, teniendo diferencias 0,15 hasta 0,62 lo cual es estadísticamente significativo. CLS fue el espacio articular de menor dimensión en todos los biotipos. Evaluando los espacios articulares por cada biotipo se encontraron diferencias significativas $(P<0,05)$ en CMS derecho, CCS derecho y muy cercano a la significancia CLS izquierdo. En todos los espacios articulares se observaron valores mayores en el sexo masculino de los cuales todos son braquifaciales siendo estadísticamente significativa $(P<0,05)$ para el espacio articular en CCS derecho, CLS Derecho y CLS Izquierdo. Conclusiones: Las dimensiones de los espacios articulares están relacionados con el biotipo facial, las medidas tomográficas de corte coronal son un insumo necesario como parte del análisis y diagnóstico anidado al biotipo facial.

Palabras clave: articulación temporomandibular; oclusión dental; biotipo facial; tomografía computarizada de haz de cono; cóndilo mandibular.

\section{Abstract}

Introduction and objective: The condylar position, as well as the facial biotype, are important to maintain an occlusion and a balanced stomatognathic system. The objective of this article is to relate the facial biotype with the condylar position in cone beam tomography in patients without temporomandibular disorders. Materials and methods: 59 Cone Beam Computer Tomography (CBCT) of 23 male and 36 female patients, with age between 14 and 59 years, were classified into dolichofacial, mesofacial and braquifacial biotypes. In order to evaluate the condylar position, the dimension of the joint spaces is evaluated. CBCT were measured with ICat vision and STATA 14 was used for statistical analysis, it was endorsed by the ethics committee of the Universidad del Valle. Results: The interobserver correlation was performed, obtaining a Kappa of 0.85 . 45 patients correspond to the braquifacial biotype, 8 dolichofacial and 6 mesofacial. In all the joint spaces, the braquifacial ones presented values of greater dimension and the dolichofacial smaller dimension. It was found that the medial spaces (CMS) present similar values in terms of laterality within each biotype, having differences of 0.02 to 0.09, however, for the central (CCS) and lateral (CLS) joint spaces greater differences between one side and the other, having differences 0.15 to 0.62 which is significant. CLS was the joint space with the smallest dimension in all biotypes. Evaluating the joint spaces for each biotype, significant differences $(P<0.05)$ were found in right $C M S$, right $C C S$ and very close to the left CLS significance. Higher values were observed in all the joint spaces in males, all of which are braquifacial, being statistically significant $(P<0.05)$ for the joint space in the right $C C S$, Right $C L S$ 
and Left CLS. Conclusions: The dimensions of the joint spaces are related to the facial biotype, the coronal section tomographic measurements are a necessary input as part of the analysis and diagnosis related to the facial biotype.

Keywords: temporomandibular joint; dental occlusion; facial biotype; cone beam computed tomography; mandibular condyle.

\section{Resumo}

Introdução e objetivo: A posição condilar, assim como o biótipo facial, são importantes para manter uma oclusão e um sistema estomatognático equilibrado. O objetivo deste artigo é relacionar o biótipo facial com a posição condilar na tomografia de feixe cônico em pacientes sem disfunção temporomandibular. Materiais e métodos: 59 tomografias de pacientes 23 do sexo masculino e 36 do feminino, com idades entre 14 e 59 anos, foram classificadas em dolicofacial, mesofacial e braquifacial. Para avaliar a posição condilar, a dimensão dos espaços articulares foi é estudada. As tomografias foram medidas com o I-Cat Vision e o STATA 14 foi usado para a análise estatística, endossada pelo comitê de ética da Universidad del Valle. Resultados: Foi realizada a correlação interobservador, obtendo-se o Kappa de 0,85. 45 pacientes correspondem ao biótipo braquifacial, 8 dolicofaciais e 6 mesofaciais. Em todos os espaços articulares, os braquifaciais apresentaram valores de maior dimensão e os dolicofaciais de menor dimensão. Verificou-se que os espaços mediais (CMS) apresentam valores semelhantes em termos de lateralidade dentro de cada biótipo, havendo diferenças de 0,02 a 0,09, porém, para os espaços articulares central (CCS) e lateral (CLS) maiores. diferenças entre um lado e o outro, tendo diferenças de 0,15 a 0,62 o que é significativo. CLS foi o espaço comum com a menor dimensão em todos os biótipos. Avaliando os espaços articulares para cada biótipo, foram encontradas diferenças significativas ( $P<0,05)$ no CMS direito, CCS direito e muito próximas à significância do CLS esquerdo. Valores mais elevados foram observados em todos os espaços articulares do sexo masculino, todos braquifaciais, sendo estatisticamente significantes $(P<0,05)$ para o espaço articular do CCS direito, CLS direito e CLS esquerdo. Conclusões: As dimensões dos espaços articulares estão relacionadas ao biótipo facial, as medidas tomográficas em corte coronal são um insumo necessário como parte da análise e diagnóstico relacionado ao biótipo facial.

Palavras-chave: articulação temporomandibular; oclusão dentária; biótipo facial; tomografia computadorizada de feixe cônico; côndilo mandibular. 


\section{Introducción}

La disciplina ortodóncica se ha mostrado históricamente interesada por las diferencias emanadas entre el diagnóstico, el tratamiento y la respuesta de la acción clínica entre los diversos biotipos faciales: mesofaciales, dolicofaciales y braquifaciales. Además, en los últimos años, un mayor número de ortodoncistas desea lograr un resultado de tratamiento en el que la posición hacia arriba y hacia adelante del cóndilo se mantenga cuando el paciente presenta la mandíbula en posición intercuspídea (PIC) ${ }^{(1)}$.

La articulación temporomandibular (ATM) está conformada por el cóndilo mandibular y la cavidad glenoidea, situada por delante del conducto auditivo externo, separados por un disco articular que define un compartimiento superior y otro inferior, los cuales no están comunicados y son lubricados por el líquido sinovial ${ }^{(2)}$. Esta articulación permite movimientos de bisagra (gínglimo) y de deslizamiento (artrodial) por lo tanto se considera ginglimoartrodial ${ }^{(3)}$.

El cóndilo mandibular se divide en cabeza y cuello. La cabeza es convexa en todos los sentidos, especialmente en sentido anteroposterior. El cuello divide la cabeza del cóndilo de la rama mandibular. La superficie superior es la que se articula con el hueso temporal y el eje longitudinal es perpendicular a la rama mandibular.

Las estructuras de la ATM, incluyendo el cóndilo mandibular, son importantes para mantener una buena oclusión y un sistema estomatognático equilibrado ${ }^{(4)}$, por lo tanto, el ortodoncista debe tener conocimiento real de la anatomía del paciente para alcanzar el correcto posicionamiento de los dientes ${ }^{(5)}$.

La ATM presenta una anatomía compleja que requiere la utilización de ayudas diagnósticas cada vez más exactas como la tomografía de haz de cono (CBCT) que permite observar de manera precisa las estructuras anatómicas ${ }^{(6)}$, esta ayuda diagnóstica tiene la ventaja de contar con una alta calidad de imagen, lo cual permite observar distintos ángulos de las estructuras anatómicas, reconstruir la ATM en tres dimensiones y evitar superposiciones de otras estructuras ${ }^{(7)}$, permitiendo un análisis con mayor exactitud de la ATM ${ }^{(8)}$. La CBTC se usa concretamente para la evaluación de los componentes óseos lo cual es ideal para la realización del presente estudio ${ }^{(9)}$. 
Un aspecto importante a tener en cuenta en el diagnóstico ortodóncico es el biotipo facial, constituyendo un carácter funcional y morfológico que determina el comportamiento de la estructura facial. Algunos autores como Steiner, Bjórk y Ricketts han descrito como evaluar el biotipo, las esferas de Bjork-Jarabak lo clasifican en tres grupos, dolicofacial si en los análisis la relación era menor a 59\%, mesofacial si estaba entre 59-63\% y braquifacial si era mayor a $63 \%{ }^{(10)}$.

La posición y la morfología condilar son características importantes para planear un tratamiento de ortodoncia, la forma y el volumen del cóndilo en adultos jóvenes desempeñan un papel importante en la estabilidad de las terapias ortodónticas y ortognáticas a largo plazo (11). Además de ellos el biotipo facial es necesario para el diagnóstico, tratamiento y respuesta terapéutica.

La posición condilar en el plano coronal ha sido previamente evaluada, en un análisis de 24 articulaciones en 22 pacientes asintomáticos entre los 12 a 25 años de edad, donde se midieron las distancias del espacio articular entre el cóndilo y la fosa glenoidea en las posiciones medial, central y lateral en el plano coronal, y en las posiciones medial y lateral en el plano axial; en el plano coronal encontraron que el espacio articular lateral era de menor dimensión que los demás espacios ${ }^{(12)}$.

Actualmente se han realizado pocos estudios que relacionen el biotipo facial con la posición o forma del cóndilo en la fosa glenoidea empleando tomografías de haz de cono en un plano sagital, en la literatura revisada del plano coronal no se encontraron artículos que relacionen estas variables. Adicionalmente la revisión no arrojó ninguno en el que la CBTC haya sido la ayuda diagnóstica de preferencia.

El presente estudio tiene como objetivo relacionar el biotipo facial con la posición condílea en tomografías de haz de cono en pacientes sin trastornos temporomandibulares, que acudieron a consulta de ortodoncia en una clínica privada en de la ciudad de Cali, Valle del Cauca.

\section{Materiales y métodos}

Se realizó un estudio de diseño descriptivo de corte transversal, con el aval del comité institucional de ética humana de la Universidad del Valle con el acta de aprobación 152-019, se llevó a cabo un muestreo no probabilístico en donde se eligieron tomografías cone-beam de cabeza y cuello pertenecientes a una base de datos del área de ortodoncia de una clínica 
privada en la ciudad de Cali, dichas imágenes fueron obtenidas con fines diagnósticos y terapéuticos se analizaron las variables edad, sexo, biotipo y posición condilar.

La información obtenida es de uso exclusivo para la investigación, por lo tanto, solo los investigadores tuvieron acceso a esta durante el estudio, evitando incumplir la normatividad de la privacidad de los participantes.

Los criterios de inclusión fueron pacientes con dentición permanente que asistieron por motivos ortodóncicos en edades entre 14-59 años, con imágenes tomográficas desde 2014 al 2019. Se excluyeron las imágenes de pacientes en cuya historia clínica se reportan antecedentes de cirugía de ATM, signos ó síntomas dolorosos de la ATM durante los últimos 6 meses contados a partir del examen inicial, así como aquellos con registro de 3 o más pérdidas de piezas dentales o que reportaron tratamiento quirúrgico de cóndilos, cirugía ortognática, trauma cráneo-buco-maxilofacial o tratamiento de ortodoncia previo. Las mediciones de las tomografías fueron desarrolladas mediante el software I-Cat Vision en un solo equipo.

El biotipo facial se determinó mediante las esferas de Bjork-Jarabak, (Figura 1) obteniendo la altura facial posterior con los puntos anatómicos de Silla (S) a Gonion (Go) y altura facial anterior de Nasion (N) a Menton (Me). Se utilizó esta fórmula con el fin de categorizar cada biotipo según el resultado.

Biotipo facial $=$ Biotipo facial $=\frac{\text { Altura facial posterior }}{\text { Altura facial anterior }} x 100$
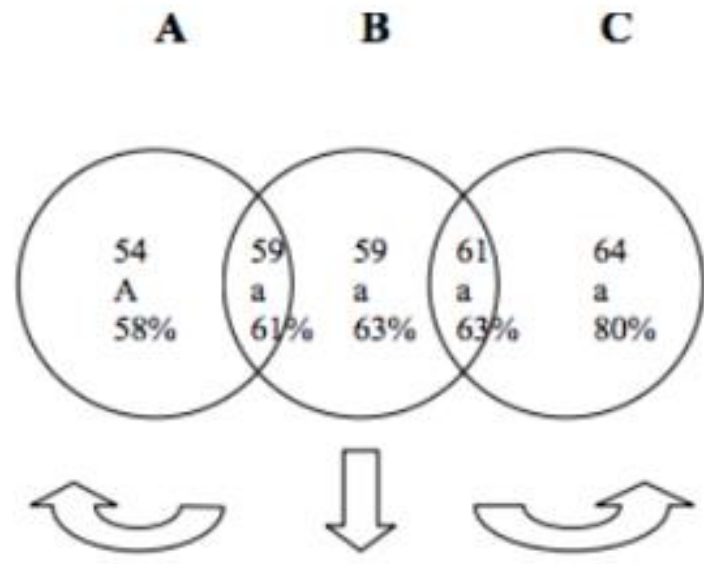

Figura 1. Biotipo facial mediante las esferas de Bjork-Jarabak. Fuente: Dra. Leticia García Vignolo; 2008. 
Con el fin de determinar la posición condilar se tomó como referencia la técnica de lkeda Kazumi dividiendo la cavidad glenoidea en sextantes, esto se realiza tomando el plano horizontal verdadero (THL) que pasa por la parte superior de la fosa glenoidea, se determinó el espacio medial (CMS) que es la tangente desde el punto medial más alto del cóndilo a la fosa glenoidea, el espacio central (CCS) corresponde a la tangente que va desde el punto más alto de la fosa glenoidea hasta el punto central del cóndilo y el espacio lateral (CLS) tangente que va desde el punto lateral más alto del cóndilo a la fosa glenoidea (igura 2).
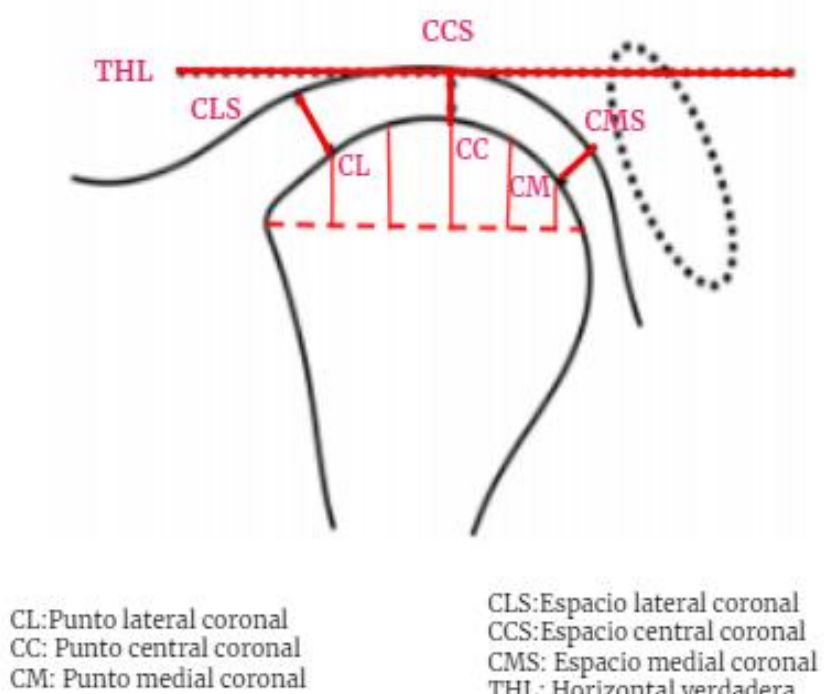

CLS:Espacio lateral coronal CCS:Espacio central coronal CMS: Espacio medial coronal THL: Horizontal verdadera

Figura 2. Posición condilar mediante la técnica de Ikeda Kazumi. Fuente: Elaboración propia.

Para el análisis de datos se utilizó el programa estadístico STATA 14. Teniendo en cuenta el tipo de variables, se obtuvieron medidas resumen para cada una de las mismas. Con el fin de garantizar la fiabilidad de las mediciones se realizó una correlación interoperador previo a la recolección de la información.

Se realizó un análisis de tipo descriptivo de las variables, frecuencia para el biotipo facial, sexo y edad, se calcularon las medidas resumen (Media y desviación estándar) para las variables en escala numérica de edad, espacio articular medial, central y lateral para cada lado. 
Se evaluó las diferencias para cada biotipo facial y respecto a los espacios articulares en cada uno de los cuadrantes y las medidas. El test de Kruskal-Wallis' y de Mann-Whitney fueron usados para determinar si existían diferencias significativas en relación con las variables del estudio biotipo y sexo.

\section{Resultados}

Se obtuvieron 59 tomografías de pacientes que cumplían todos los criterios de inclusión y exclusión, la correlación interoperador obtuvo un Kappa de 0,85, siendo adecuado para continuar con la toma de las medidas.

La edad de los pacientes se encontró entre los 14 y 59 años con un promedio de edad de 28,11 $( \pm 12,44)$. El $61,02 \%$ corresponde al sexo femenino y el $38,98 \%$ al sexo masculino de la muestra obtenidas (Tabla 1).

De las 59 imágenes tomográficas el 76,27 \% corresponde a pacientes con biotipo braquifacial, el $13,56 \%$ a pacientes con biotipo mesofacial y solo el $10,17 \%$ a pacientes con el biotipo dolicofacial (Tabla 1).

Tabla 1. Características sociodemográficas.

\begin{tabular}{lccc}
\hline \multirow{4}{*}{ Edad } & & \multicolumn{1}{c}{$\boldsymbol{N}$} & $\%$ \\
\hline \multirow{3}{*}{ Sexo } & $14-59$ & 19 & 32,20 \\
\cline { 2 - 4 } & $20-29$ & 16 & 27,12 \\
\cline { 2 - 4 } & $20-39$ & 13 & 22,03 \\
\cline { 2 - 4 } & 40 y más & 11 & 18,64 \\
\cline { 2 - 4 } Biotipo & Masculino & 23 & 38,98 \\
\cline { 2 - 4 } & Femenino & 36 & 61,02 \\
\cline { 2 - 4 } & Braquifacial & 45 & 76,27 \\
\cline { 2 - 4 } & Dolicofacial & 6 & 10,17 \\
\hline & Mesofacial & 8 & 13,56 \\
\hline
\end{tabular}


El espacio articular lateral es de menor tamaño que el central y medial tanto en el lado derecho como en el izquierdo (Tabla 2).

Tabla 2. Promedio de las dimensiones de los espacios articulares según el lado.

\begin{tabular}{lcc}
\hline Espacio articular & Derecho media \pm de & Izquierd media \pm de \\
\hline Medial coronal (CMS) & $2,69 \pm 0,94$ & $2,69 \pm 1,01$ \\
\hline Central coronal (CCS) & $2,61 \pm 0,90$ & $2,62 \pm 0,93$ \\
\hline Lateral coronal (CLS) & $2,24 \pm 0,81$ & $2,39 \pm 1,04$ \\
\hline
\end{tabular}

Los espacios articulares mediales presentaron valores similares en cuanto a lateralidad dentro de cada biotipo siendo valores más pequeños en los dolicofaciales sin embargo para los espacios centrales y laterales se observaron mayores diferencias entre un lado y otro en todos los biotipos, también se observó que todos los espacios articulares a excepción del CLS izquierdo en el dolicofacial fueron valores menores que para los otros biotipos faciales. Todos los espacios articulares en los braquifaciales presentaron mayor tamaño que en los otros biotipos, la P de significancia establecida por el test de Kruskall Wallis muestra diferencia entre al menos un grupo de los biotipos para CMS Derecho, CCS Derecho y muy cercano a la significancia CLS Izquierdo (Tabla 3 ).

Tabla 3. Promedio de las dimensiones de los espacios articulares según el lado y el biotipo facial.

\begin{tabular}{|c|c|c|c|c|}
\hline & Braquifacial & Dolicofacial & Mesofacial & $P^{*}$ \\
\hline Espacio articular & Media \pm de & Media \pm de & Media \pm de & \\
\hline Medial coronal (CMS) derecho & $2,83 \pm 0,91$ & $1,86 \pm 0,82$ & $2,50 \pm 0,96$ & 0,05 \\
\hline Medial coronal (CMS) izquierdo & $2,81 \pm 1,00$ & $1,95 \pm 0,87$ & $2,53 \pm 1,02$ & 0,21 \\
\hline Central coronal (CCS) derecho & $2,85 \pm 0,86$ & $1,64 \pm 0,46$ & $2,03 \pm 0,66$ & 0,00 \\
\hline Central coronal (CCS) izquierdo & $2,67 \pm 0,96$ & $2,16 \pm 1,43$ & $2,65 \pm 0,91$ & 0,14 \\
\hline Lateral coronal (CLS) derecho & $2,34 \pm 0,77$ & $1,60 \pm 0,83$ & $2,11 \pm 0,89$ & 0,18 \\
\hline Lateral coronal (CLS) izquierdo & $2,49 \pm 0,84$ & $2,50 \pm 2,17$ & $1,77 \pm 0,86$ & 0,07 \\
\hline
\end{tabular}

*Prueba Kruskall Wallis. 
Tanto en el lado derecho como en el izquierdo se observa que los espacios articulares de los hombres son mayores comparado con las mujeres, el test de Wilcoxon Mann whitney muestra una diferencia estadísticamente significativa para el espacio articular en CCS derecho, CLS Derecho y CLS Izquierdo (Tabla 4).

Tabla 4. Promedio de las dimensiones de los espacios articulares según el sexo.

\begin{tabular}{llll}
\hline & Masculino & Femenino & $\boldsymbol{P}^{*}$ \\
\hline Espacio articular & Media \pm de & Media \pm de & \\
\hline Medial coronal (CMS) derecho & $2,92 \pm 0,86$ & $2,54 \pm 0,98$ & 0,10 \\
\hline Medial coronal (CMS) izquierdo & $2,96 \pm 0,97$ & $2,51 \pm 1,02$ & 0,10 \\
\hline Central coronal (CCS) derecho & $3,01 \pm 0,86$ & $2,36 \pm 0,84$ & 0,00 \\
\hline Central coronal (CCS) izquierdo & $2,82 \pm 0,95$ & $2,49 \pm 0,90$ & 0,17 \\
\hline Lateral coronal (CLS) derecho & $2,62 \pm 0,63$ & $1,99 \pm 0,82$ & 0,00 \\
\hline Lateral coronal (CLS) izquierdo & $2,71 \pm 0,80$ & $2,19 \pm 1,14$ & 0,00 \\
\hline
\end{tabular}

*Test de Wilcoxon Mann-whitney.

\section{Discusión}

El espacio articular presenta variaciones de acuerdo con el biotipo facial ya que se observaron diferencias significativas en algunas de las medidas, en números absolutos se pudo evidenciar que los dolicofaciales presentan menor dimensión en los espacios articulares y los braquifaciales mayor dimensión.

Este estudio logró establecer que los espacios articulares dependen del biotipo por lo cual al analizar una tomografía en un corte coronal se debe tener en cuenta que las dimensiones del espacio articular depende del biotipo facial, además que existe una diferencia en sexo, siendo en números absolutos más extensas las medidas en hombres que en mujeres y teniendo significancia estadística en 3 de ellas. 
En la literatura revisada no se encontraron estudios que relacionen estas variables, sin embargo se intentó determinar el volumen condilar en sujetos con diferentes patrones esqueléticos anteroposterior y vertical, se observó que un mayor volumen condilar era una característica común de los sujetos con hipodivergencia (braquifacial) en comparación con los sujetos con normodivergencia (mesofacial) e hiperdivergencia (dolicofacial) ${ }^{(13)}$, En este estudio los espacios articulares fueron mayores en los braquifaciales por lo que sugiere que el grosor del disco articular y del tejido conectivo son mayores lo que se relacionaría con el volumen condilar mayor.

Se encontró similitud frente al estudio que evaluó la posición condilar en el plano coronal con una muestra de 22 pacientes de origen japonés en donde se midieron los espacios articulares en plano axial y coronal ${ }^{(9)}$, en cuanto al espacio articular lateral que es de menor tamaño que el central y medial, sin embargo, el autor del artículo no hace referencia al biotipo ni diferencia la lateralidad en sus resultados.

En todos los espacios articulares se observaron valores mayores en el sexo masculino, estos representan 23 pacientes de los cuales todos son braquifaciales, sin embargo, no se encuentran estudios que diferencien un biotipo para cada sexo, y en el estudio de Kazumi ${ }^{(12)}$, se evalúo la posición condilar pero no se encontraron diferencias significativas de los espacios articulares entre el sexo.

El patrón facial vertical influye en la fuerza oclusal máxima y en las actividades musculares masticatorias ${ }^{(14)}$. Existe una mayor prevalencia de trastornos temporomandibulares (TTM) en pacientes con el patrón esquelético dolicofacial más que en los braquifaciales (15). El desplazamiento discal sin reducción está asociado a una pronunciada distalización condilar y disminución del espacio articular ${ }^{(16)}$; con las tomografías analizadas se observó que el espacio articular presenta variaciones de acuerdo al biotipo facial, demostrando que el biotipo dolicofacial presenta una disminución en las dimensiones de los espacios articulares, por lo cual concuerda que los TTM pueden ser más frecuentes en estos pacientes debido a la disminución de los espacios articulares.

Dentro del análisis y el proceso de revisión, este es el único estudio en Colombia que propone una diferenciación del biotipo facial en relación con la posición condilar, el entender este aspecto será de gran utilidad para definir acciones orientadas a los ortodoncistas para su 
proceso diagnóstico y de tratamiento y además para fortalecer el uso de la CBTC como fuente diagnóstica de elección y con la posibilidad de profundidad en los diagnósticos mucho más acertados.

El estudio hizo evidente la relación que existe entre las variables biotipo y las específicas de la posición condilar y que deben tenerse en cuenta al iniciar un tratamiento puesto que no se encontraran las mismas dimensiones de los espacios articulares en diferentes biotipos, esto también influye en el movimiento de piezas dentales ya que estos se ven reflejados en la posición del cóndilo articular.

Una de las limitaciones fue la asimetría de la muestra por lo que se sugiere que para futuros estudios se incremente la población dolicofacial y mesofacial obteniendo una muestra más simétrica, conservando las diferencias de las prevalencias que perse se conocen de las mismas y que dependen del contexto de desarrollo del estudio.

El biotipo facial y la posición condilar no se han evaluado en gran medida especialmente en el plano coronal, ya que en la mayoría de los casos se evalúa la posición sagital del cóndilo, por lo tanto, este estudio es de gran relevancia clínica aportando conocimiento innovador y que pueden servir de guía para futuras investigaciones o para el clínico mismo.

Como parte del desarrollo de esta línea de investigación, se propone nuevos estudios que comparen las variables de este estudio con los trastornos temporomandibulares y así poder tener una correlación de las implicaciones clínicas, además se requieren muestreos mayores que implique la inclusión de población diversa, incluso bajo una mirada pluriétnica y multicultural, tal cual como la presenta el país en la actualidad.

\section{Conclusiones}

El analisis realizado pudo establecer que existe una diferencia en sexo siendo los espacios articulares de los hombres mayor que las mujeres. Además que los pacientes dolicofaciales presentan menor dimensión en los espacios articulares y los braquifaciales mayor dimensión de los mismos.

El presente estudio demostró la relación existente entre los espacios articulares y el biotipo facial, lo que evidencia que la tomografía de corte coronal que es un insumo diagnóstico de gran importancia en la disciplina odontológica, es muy útil para definir las mediciones y como medio diagnóstico y de seguimiento a los tratamientos ortodóncicos. 


\section{Referencias}

1. Girardot A. Comparison of Condylar Position in Hyperdivergent and Hypodivergent Facial Skeletal Types. The Angle Orthodontist; 2001; 71 (4): 240-246.

2. Concha Guillermo, Imágenes por resonancia magnética de la articulación temporomandibular. Revista HCUCh; 2007; (18): 121 - 30.

3. Okeson J. Tratamiento de oclusión y afecciones temporomandibulares. 5th ed. Madrid, España: Elsevier; 2013: 8-26.

4. Arieta. J, Silva. M, Flores.C,Paredes.N. Spatial analysis of condyle position according to sagittal skeletal relationship, assessed by cone beam computed tomography. Progress in Orthodontics; 2013; 14: 36.

5. Roque Gina D, Meneses Abraham, Norberto Frab, De Almeida Solange, Haiter Francisco. La tomografía computarizada cone beam en la ortodoncia, ortopedia facial y funcional. Rev. Estomatol. Herediana; 2015; 25, (1): 61-78.

6. Finlayson Antonio F.Epifanio Rodolfo.La tomografía computarizada de haz cónico.Revista UstaSalud; 2008; 7(2): 125-131.

7. Moya Juan Pablo, Nasu Steven, Martinez Christian. Conceptos fundamentales en la interpretación de la tomografía de radio de cono desde la odontología general. Grupo insao, Universidad Autónoma de Manizales; 2009.

8. Park, I. Y., Kim, J. H., \& Park, Y. H. Three-dimensional cone-beam computed tomography based comparison of condylar position and morphology according to the vertical skeletal pattern. Korean journal of orthodontics; 2015; 45(2): 66-73.

9. Gorucu-Coskuner H, Atik E, El H. Reliability of cone-beam computed tomography for temporomandibular joint analysis. Korean J Orthod; 2019; 49(2): 81-88.

10. Cerda-Peralta Bárbara, Schulz-Rosales Rolando, López-Garrido Jimena, RomoOrmazabal Fernando. Cephalometric norms related to Facial type in eugnathic Chilean adults. Rev. Clin. Periodoncia Implantol. Rehabil. Oral; 2019; 12 (1): 8-11.

11. Saccucci M, Polimeni A, Festa F, Tecco S. Do skeletal cephalometric characteristics correlate with condylar volume. surface and shape? A 3D analysis. Head Face Med; 2012; 8: 15. 
12. Kazumi Ikeda, Kawamura A. Assessment of Optimal Condylar Position in in the Coronal and Axial Planes with Limited Cone-Beam Computed Tomography. Journal of Prosthodontics; 2011; 20: 432-438.

13. Hasebe. A, Yamaguchi.T, Nakawaki.T, Hikita Y. Comparison of condylar size among different anteroposterior and vertical skeletal patterns using cone-beam computed tomography. The Angle Orthodontist; 2019; 89; (2): 306-311.

14. Custodio W, Gomes SG, Faot F, Garcia RC, Del Bel Cury AA. Occlusal force, electromyographic activity of masticatory muscles and mandibular flexure of subjects with different facial types. J Appl Oral Sci; 2011; 19: 343-349.

15. Stringert HG, Worms FW. Variations in skeletal and dental patterns in patients with structural and functional alterations of the temporomandibular joint: a preliminary report. Am J Orthod; 1986; 89: 285-297.

16. Maglione, Horacio. Disfunción craneomandibular. Afecciones de los músculos masticadores y de la ATM, dolor orofacial; 2008. 